\title{
4 Design of Application Software for Setting Technological Parameters and Briquette Quality Estimation According to the Configured Parameters
}

To design a simple mathematical model that is also user-friendly, it was necessary to design a user friendly tool that the model would use. To fulfill this requirement, simple application software was created to function based on the derived mathematical model. The basic requirements for the software include: an overview of the environment, user-friendliness, report generation, and an easy interface with a user's manual.

The application software was designed in DELPHI and with minimal hardware requirements for using the application software (running on Windows 95 or 98, $128 \mathrm{MB}$ of memory (RAM) and 5MB of free disk space required). The designed application software operates under Windows XP or Windows 7 with no problem. The user environment for the application software with basic descriptions can be seen in the Figures 4.1 and 4.2. The software environment constitutes several basic parts (Fig. 4.2).

Component part description of the application software:

1. Application control- contains options such as "start"(after entering inputs, the function starts analysis), "print protocol”(prints protocol), "Quit”(quits the application software), and basic information about the application software.

2. Material configuration - enables the selection of input material. Currently, the configuration is limited only to pine. As previously described, this raw material was used in the enclosed experiments and the foundation to which the mathematical model was constructed. Expansion of this mathematical model to include spruce, oak, beech, and other wood materials has been planned.

3. Entering input parameters - enables the entrance of input values of the examined parameters in listed units. In this part of the interface, the desired density value of the briquette is displayed after running the analysis. The values of each of the parameters can be selected only from intervals, which are listed in the information part.

4. Information part - here, the value intervals for each of the examined parameters, from which the user can select, are listed. Momentarily, the listed intervals of values are those which are valid for the mathematical mode and for the executed experiment. In the future, the interval will be expanded.

5. Error messages- warns the user about incorrect input values. For example, if the input value is outside the interval range, the density cannot be calculated.

6. Illustrative display of the entering fraction (sawdust) and product. Interestingly, the pictures change according to the configured value for the fraction size.

7. Illustrative display of the experimental press stand with the heating device. 


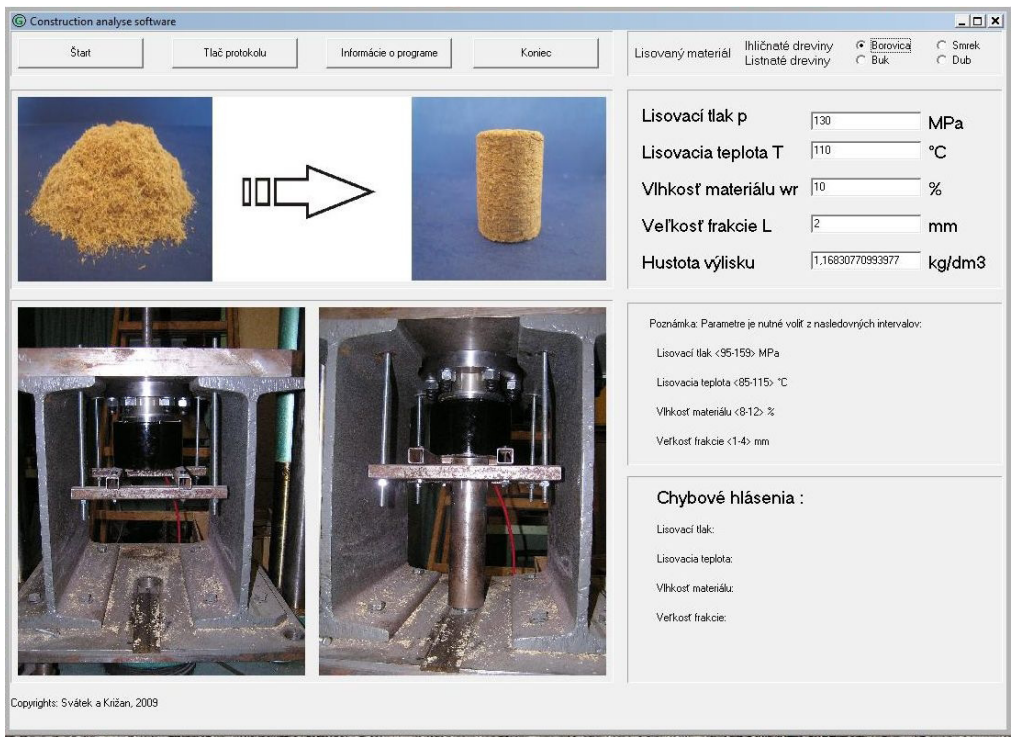

Figure 4.1: Application software view (Križan, 2009).

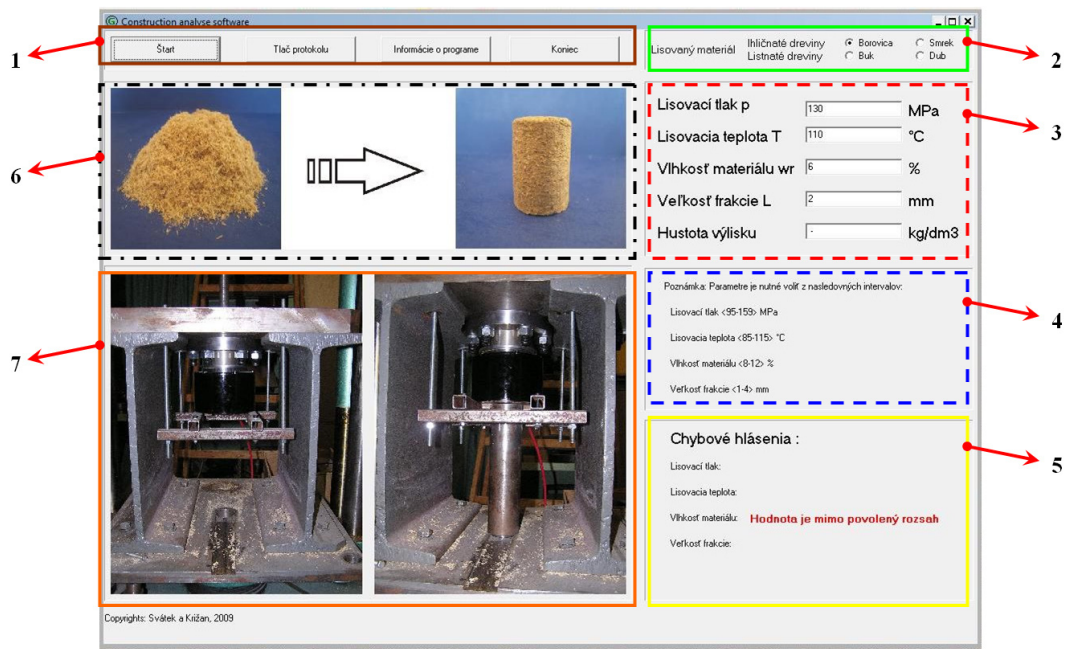

Figure 4.2: Basic overview of the designed software (Križan, 2009). 
The designed application software can operate in two basic modes. In the first mode, the values of the examined parameters (pressing pressure, pressing temperature, moisture content of the material, and the size of the raw material input fraction) are configured, and the application displays the estimation of the resulting density values after the analysis.

In the second mode, the application functions in a reversed manner. The density values of the briquette, along with three or four of the examined parameters, are set. The application will display the estimates for the remaining parameters after the analysis. The application software can be a useful tool for estimating the quality of briquettes according to the configured parameters or for optimizing structural parameters.

In the first case for using this software, the application can be a useful tool for briquette producers. It is estimated that most producers typically use pressing machines on which it is not possible to change the pressing pressure. This parameter is constant and is one of the input parameters. On this application, the producer can also enter the pressing temperature (depending on the pressing machine, control may or may not be possible), the moisture content of the input fraction, and its size. After analysis with the aid of the designed application, the user receives the estimated values for the density of the briquette before commencing the process itself. Subsequently, the user can use the application for predicting the values for examined parameters. Another possible input value is the briquette density that the user wishes to achieve. If the producer uses a pressing machine on which it is not possible to control the pressing temperature, then these parameters can be constant, fixed input as well. However, if the temperature can be regulated, the value must be set. Material parameters (material humidity and fraction size) can be easily obtained according to the technology requirements. In summary, there are 2-3 fixed inputs and 2-3 controllable inputs. The application is able to control the parameters and estimate optimal parameters for the pressing process to achieve the desired density.

The second case for using the designed application is the use of the software as a tool for optimizing technology parameters. Pressing machine constructors can use this application for predicting the pressing pressure and temperature. By setting fixed values for the briquette density and material parameters (moisture content and fraction size), the application is able to gradually regulate the pressing pressure and temperature and estimate the necessary values for these parameters. These optimal values are then the input values when designing the construction and structural analysis of the pressing chamber, pressing tool, and drive that facilitate the pressing process.

Using this application, blueprints for creating illustrative graphical dependencies of the density from the examined parameters (pressing press, material moisture content, and fraction size) can be found for several temperature levels. These dependencies are shown and described in Figures 4.3-4.5. It is necessary to understand that the listed dependencies and examples for using the application software are 
valid for pressing pine sawdust only. Expanding the usability of this application for other types of materials and expanding the interval values requires conducting more experiments. The results from these experiments would alter the mathematical model, and thus the designed application using this model would need modification.

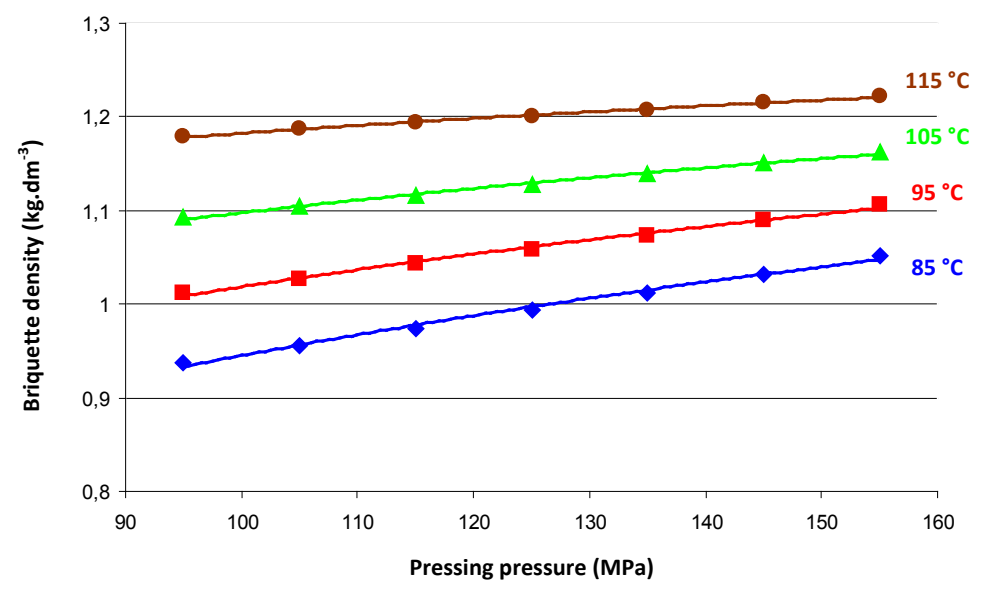

Figure 4.3: Briquette density dependence with respect to various pressing temperatures for pine sawdust $\left(w_{r}=10 \% ; L=2 m m\right)$ ( Križan, Šooš \& Vukelić, 2009; Križan \& Matúš, 2008).

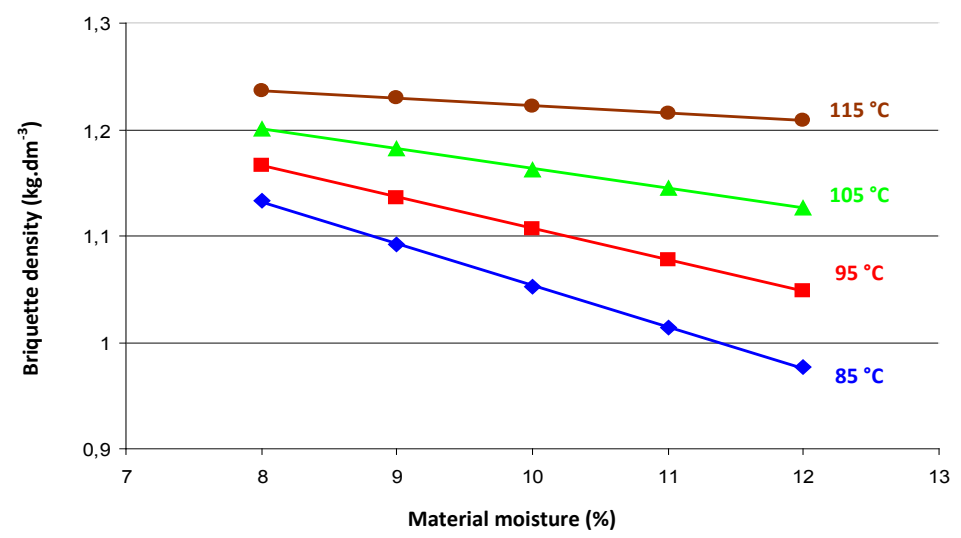

Figure 4.4: Briquette density dependence with respect to material moisture at various pressing temperatures for pine sawdust ( $p=155 \mathrm{MPa}$; $=2 \mathrm{~mm}$ ) (Križan, Šooš \& Vukelić, 2009; Križan \& Matúš, 2008). 


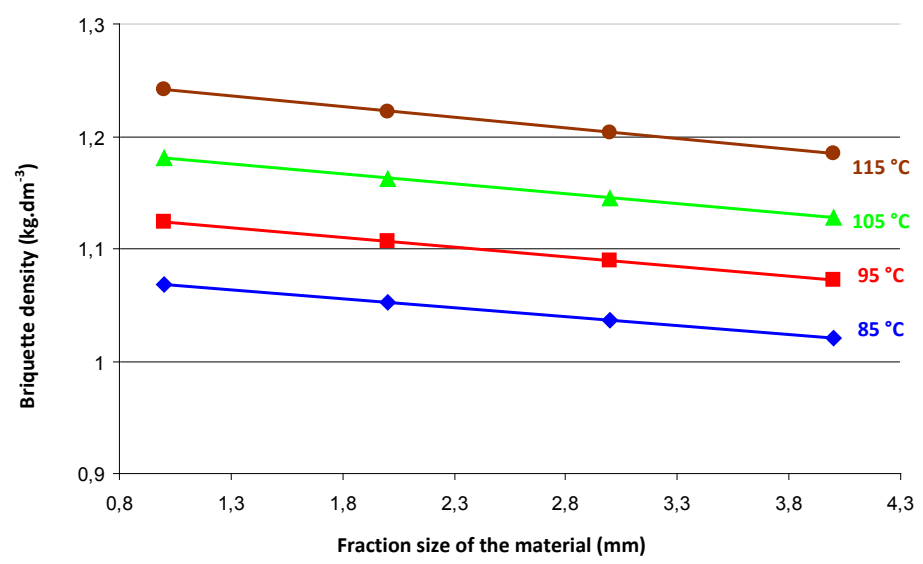

Figure 4.5: Briquette density with respect to the material fraction size at various pressing temperatures, for pine sawdust $\left(w_{r}=10 \% ; p=155 \mathrm{MPa}\right)$ ( Križan, Šooš \& Vukelić, 2009; Križan \& Matúš, 2008).

Based on the dependencies shown, the four examined parameters have a large impact on the quality of the briquette as evaluated by the resultant briquette density during the pressing process. Fig. 4.3 shows that the briquette density increases with increasing pressing pressure and temperature. This dependency shows a change in briquette densities at pressing pressures from 95 to $159 \mathrm{MPa}$ and in the temperature interval from 85 to $115^{\circ} \mathrm{C}$. The pressing temperature and pressure are parameters that mutually affect each other and thus affect the resultant density. At a given pressing temperature, it is necessary to obtain a briquette with a certain density at a lower pressure. The dependency shown in Fig. 4.3 is a depiction of this principle. Consequently, pressing machine builders must either strive for lower pressing pressures at higher pressing temperatures in their designs, or vice versa. However, the pressing temperature has a larger impact on density than the pressing pressure per the present study's findings, and so we recommend that pressing machines should be constructed with a focus on regulating temperature.

Fig. 4.4 shows the dependence between briquette density and moisture content of the raw material at various pressing temperatures is. The dependency shows a change in briquette density in a range of material water content from 8 to $12 \%$ and in an interval of pressing temperatures from 85 to $115^{\circ} \mathrm{C}$. The dependency shows how the moisture content of the material combined with pressing temperature affects the resultant briquette density. We can see that, with decreasing values of water content and increasing pressing temperature, the briquette density increases. This results in better plasticisation of the lignin within the material at higher temperatures and smoother connections between the plasticized lignin and the material particles when under pressure. The dependency seen in Fig. 4.4 is different from that in Fig. 2.40. It 
is necessary to note that the experimental results are valid within an interval of 8 to $12 \%$. More experiments are needed to increase and expand this interval.

The effect of the fraction size combined with the change in pressing temperature on the resultant briquette density is shown in Fig. 4.5. The dependency shows the change in briquette density with fraction sizes from 1 to $4 \mathrm{~mm}$ and an interval of pressing temperature from 85 to $115{ }^{\circ} \mathrm{C}$. We can see that, with a decreasing size of fraction and increasing pressing temperature, the briquette density increases. Small fractions obtain a better bond amongst the material particles and the lignin when solid particles in the material contact each other. Thus, there is more contact area and number of atoms in the interphases between particles. Decreasing the size of the fraction shrinks the pores between solid particles and thus restricts the motion of the particles.

Thanks to the derived mathematical model, we are able to describe the effects of parameters and their mutual interactions in the pressing chamber. Another goal of this study was to define the optimal configuration of these parameters to obtain a high-quality briquette. To identify optimal configurations, density calculations using the derived mathematical model were performed for each parameter setting under different conditions (various parameter settings). This experimental study showed the most suitable as well as least suitable configurations within a given interval. These results created an applicable boundary interval for the use of the mathematical model for each monitored (affecting) parameter. Definitive values of the boundary settings can be observed in the following graphs. These graphs illustrate the applicable region that the mathematical model uses.

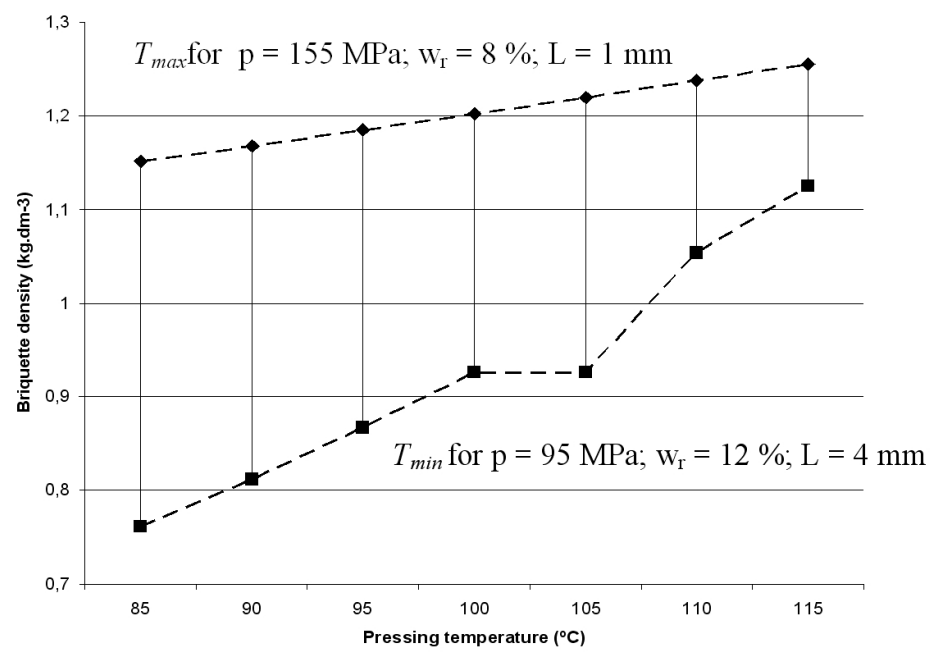

Figure 4.6: Briquette density for various settings of pressing temperature (Križan \& et al., 2010). 


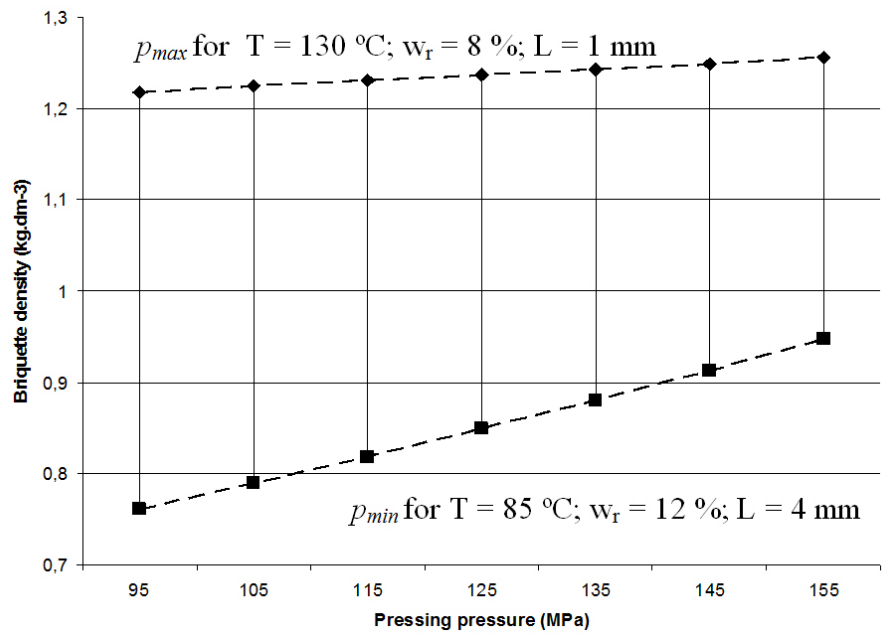

Figure 4.7: Briquette density for various pressure settings (Križan \& et al., 2010).

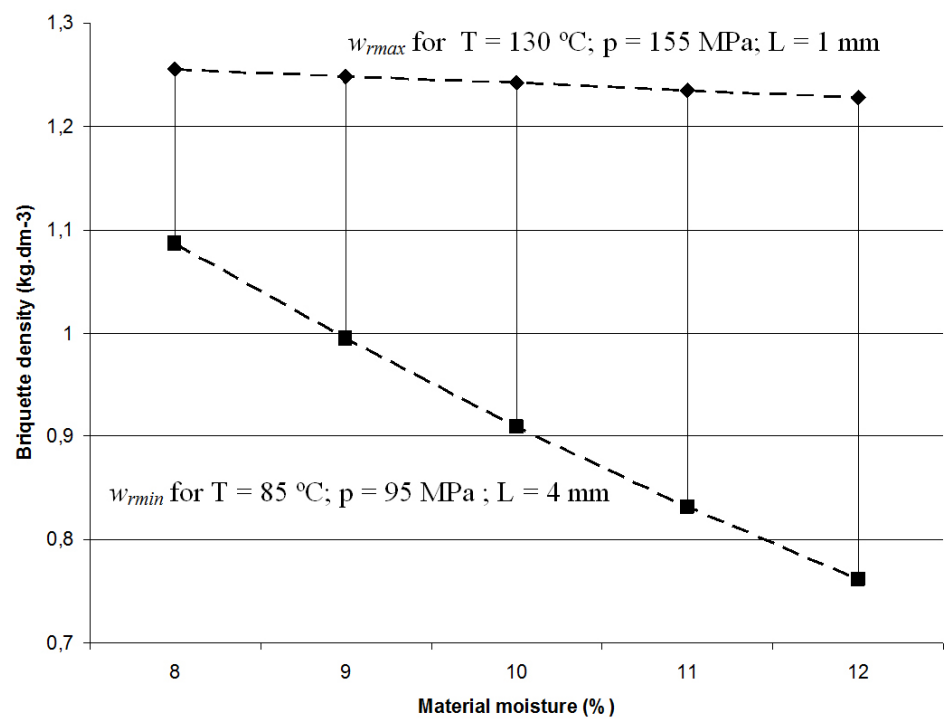

Figure 4.8: Density of briquette for various settings of input fraction moisture content (Križan \& et al., 2010). 


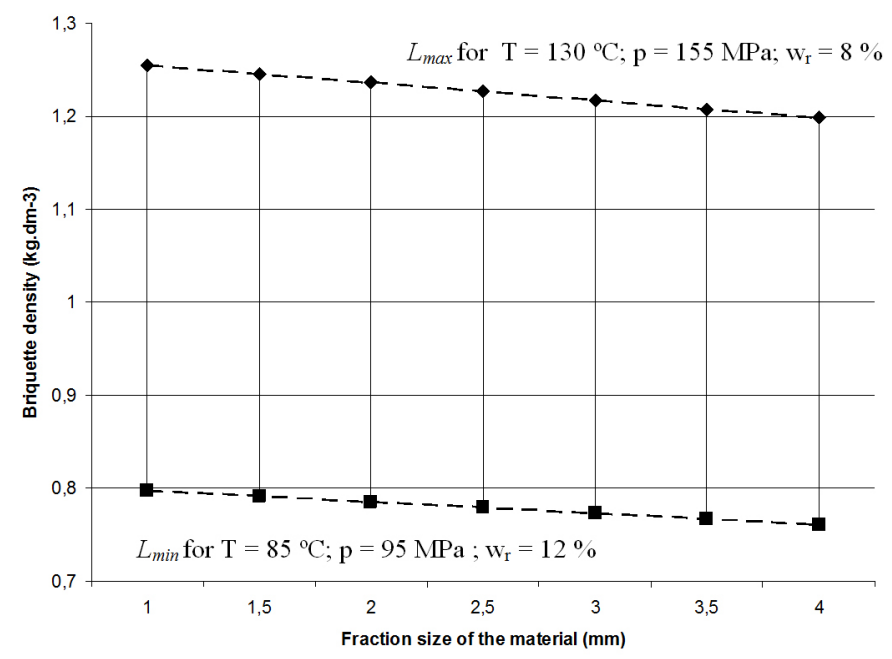

Figure 4.9: Briquette density for various settings of input fraction size (Križan \& et al., 2010).

The goal of this experimental research was to provide a tool for the accurate estimate of briquette quality with respect to some set parameters through a mathematical model. When pressing any kind of material in specific conditions, it is possible to obtain an optimal setting of the effecting parameters. Since the most important factor that defines the quality of compacted wood biomass is the briquette density, this parameter was set as the criterion for the affecting parameters. Using the mathematical model derived in this study, it is possible to define regions of optimal parameters that yield a high-quality briquette according to DIN Plus. This certification scheme was chosen because it has the most strict density values $\left(1.12 \mathrm{~kg} . \mathrm{dm}^{-3}\right)$ for wood material briquettes. The following figure illustrates where to find a specific region. This figure was created using individual sets of monitored parameters. Each set is defined by a closed interval for which an experiment was performed. These intervals are identical to those calculated by the mathematical model. 
Region of optimal parameters settings for creating high-quality briquette according to DIN Plus Standards

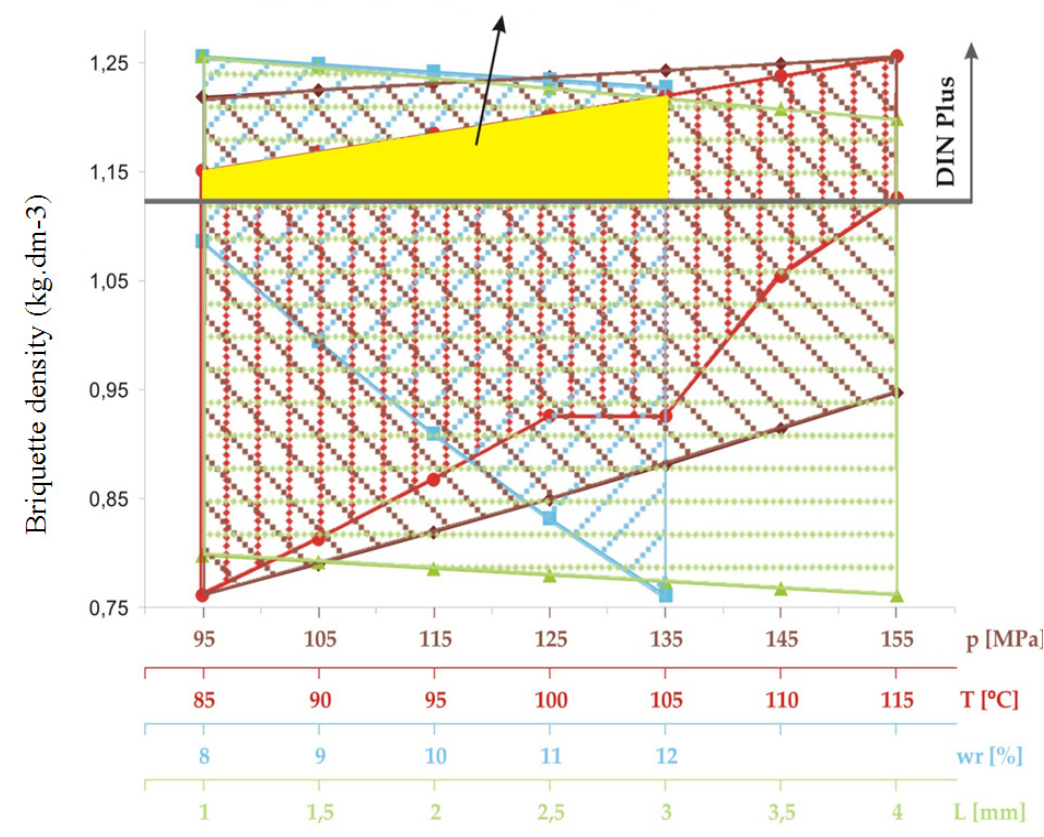

Figure 4.10: Illustrative representation showing regions of optimal parameters settings with respect to briquette quality (Križan \& Svátek 2007; Svátek \& Križan, 2013). 